\title{
An H I Census of Loose Groups of Galaxies
}

\author{
D.J. Pisano
}

NSF Distinguished International Postdoctoral Research Fellow, ATNF, P.O. Box 76, Epping NSW 1710, Australia

David G. Barnes

School of Physics, Univ. of Melbourne, Victoria 3010, Australia

Brad K. Gibson

Swinburne University of Technology, Centre for Astrophysics and Supercomputing, Mail \# 31, , P.O. Box 218, Hawthorn, Victoria 3122, Australia

Lister Staveley-Smith

ATNF, P.O. Box 76, Epping NSW 1710, Australia

Ken C. Freeman

RSAA, Mount Stromlo Observatory, Cotter Road, Weston, ACT 2611, Australia

\begin{abstract}
We present results from our Parkes Multibeam H I survey of 3 loose groups of galaxies that are analogous to the Local Group. This is a survey of groups containing only spiral galaxies with mean separations of a few hundred $\mathrm{kpc}$, and total areas of approximately $1 \mathrm{Mpc}^{2}$; groups similar to our own Local Group. We present a census of the H I-rich objects in these groups down to a $1 \sigma M_{H I}$ sensitivity $\sim 7 \times 10^{5} M_{\odot}$, as well as the detailed properties of these detections from follow-up Compact Array observations. We found 7 new H I-rich members in the 3 groups, all of which have stellar counterparts and are, therefore, typical dwarf galaxies. The ratio of low-mass to high-mass gas-rich galaxies in these groups is less than in the Local Group meaning that the "missing satellite" problem is not unique. No high-velocity cloud analogs were found in any of the groups. If HVCs in these groups are the same as in the Local Group, this implies that HVCs must be located within $\sim 300-400 \mathrm{kpc}$ of the Milky Way.
\end{abstract}

\section{Introduction}

Loose groups of galaxies are collections of a few (2-3) large galaxies and tens of smaller galaxies. They are the most diffuse components of structure in the universe, yet they are relatively understudied despite their importance. About $60 \%$ 
of galaxies reside in groups (Tully 1987) including the Milky Way which is part of the Local Group of galaxies. Loose groups are possibly still forming (Zabludoff \& Mulchaey 1998), and may even be the site of ongoing galaxy formation as traced by the high-velocity clouds (HVCs; e.g. Blitz et al. 1999). Measuring the distribution of galaxy masses in groups also provides a useful constraint on models of galaxy formation. To learn more about groups of galaxies similar to our own, to constrain the nature of HVCs, and to learn more about galaxy and structure formation, we have commenced a survey of loose groups of galaxies.

\section{Observations}

Our survey examined analogs to the Local Group: spiral-rich loose groups without any large elliptical or lenticular galaxies. We selected five such groups from the LGG catalog of Garcia (1993). A sixth group was selected from the HIPASS group catalog (Stevens, 2003, private communication) and was not previously identified optically. Assuming $\mathrm{H}_{0}=65 \mathrm{~km} \mathrm{~s}^{-1} \mathrm{Mpc}^{-1}$, the groups lie between 10.6 - 13.4 Mpc. We observed a projected area of $1-1.7 \mathrm{Mpc}^{2}$ centered on each group using the Parkes Multibeam receiver. The observations involved scanning the instrument in a "basket-weave" pattern in right ascension and declination multiple times until an RMS sensitivity of $\sim 7 \times 10^{5} M_{\odot}$ per $3.3 \mathrm{~km} \mathrm{~s} \mathrm{~s}^{-1}$ was reached. The total velocity coverage of the observations was either 1700 or $3400 \mathrm{~km} \mathrm{~s}^{-1}$ (depending on the group) with a velocity resolution of $1.65 \mathrm{~km} \mathrm{~s}^{-1}$ or $3.3 \mathrm{~km} \mathrm{~s}^{-1}$. The linear resolution of the Parkes observations was $\sim 50 \mathrm{kpc}$.

The data were reduced and gridded into cubes using the ATNF livedata and gridzilla packages in aips ++ . The final cubes were searched by eye by three people for detections. Fake sources were inserted into these cubes to assess the reliability and completeness of the search. If a source was found by at least 2 people, it was considered to be a detection. Based on this analysis, our detection algorithm was essentially $100 \%$ complete down to the $10 \sigma$ level.

All our detections, not just the new ones, were re-observed with the ATCA. The ATCA observations not only served to confirm the detections, but the higher spatial resolution, $\sim 4 \mathrm{kpc}$, allowed us to uniquely identify $\mathrm{H}$ I detections with optical counterparts. Furthermore, as the ATCA observations had about the same sensitivity as the Parkes cubes, we were able to search for $\mathrm{H}$ I clouds which were previously unresolved in the Parkes data.

The combination of the large area observed, high velocity resolution, and extremely sensitive observations of multiple loose groups makes this survey uniquely tuned to get a census of the H I content of loose groups of galaxies and to strongly constrain the origin of HVCs. We will address these topics using the first 3 groups studied in our survey: LGG 93, LGG 180, and LGG 478.

\section{The H I Content of Loose Groups of Galaxies}

Cold dark matter simulations of the formation of the Local Group of galaxies (e.g. Klypin et al. 1999, Moore et al. 1999) reveal an extremely large number of small dark matter halos ( 300) compared to the number of known luminous satellite galaxies $(\sim 20)$. This is the so-called "missing satellite" problem. While invoking a different type of dark matter, such as warm dark matter, or invoking 


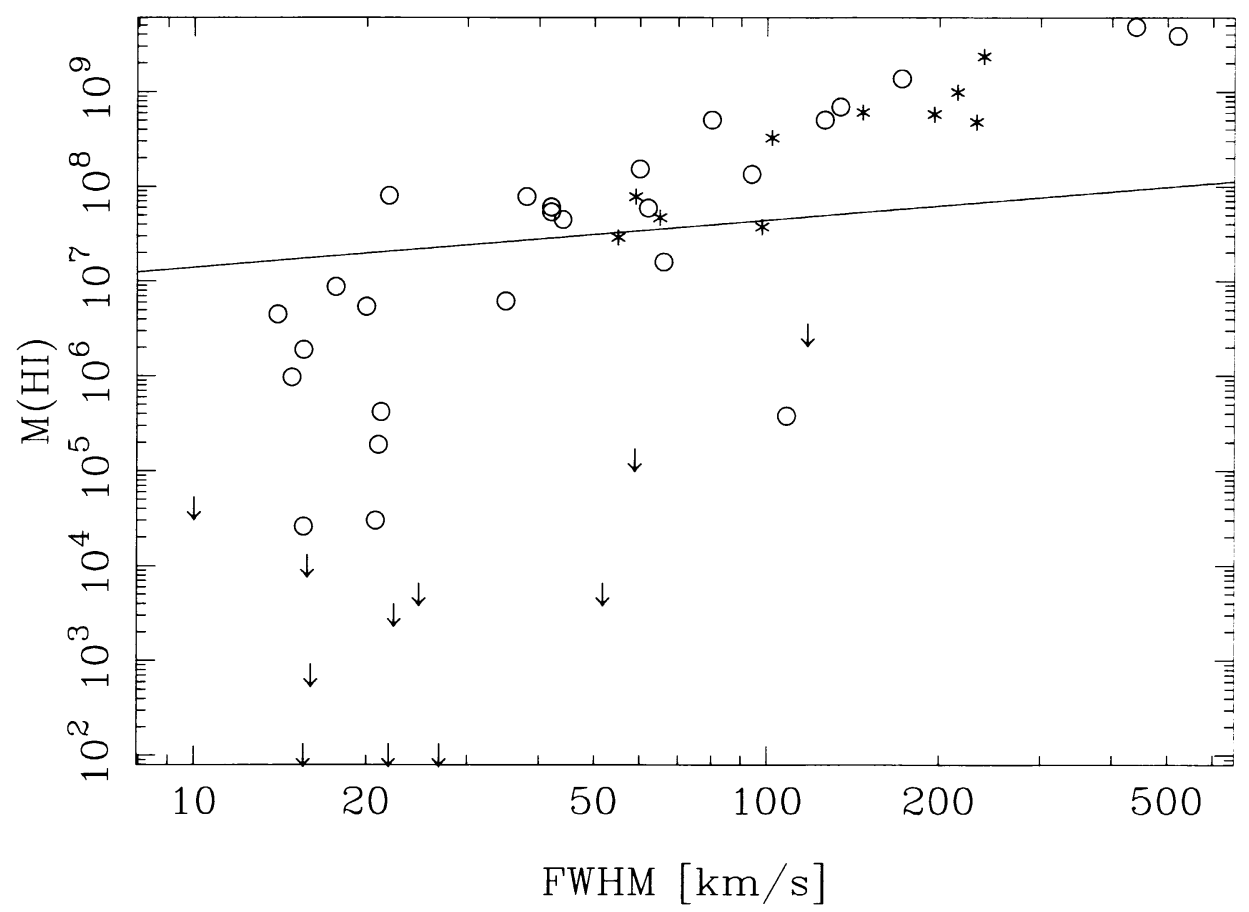

Figure 1. $M_{H I}$ vs. $\mathrm{V}_{\text {rot }}$ for Local Group galaxies (from Mateo 1998) with measured $M_{H I}$ (circles) and upper limits (arrows). The stars indicate the sources in LGG 93. The line indicates the $10 \sigma$ detection limit

feedback processes to suppress the collapse of baryons in dark halos can solve this problem, it is worth asking if this problem exists at the same level in groups similar to the Local Group.

Our survey detected all the optically identified galaxies in the 3 groups. In addition, we detected 4 new group members in LGG 93, 2 new group members in LGG 180, and 1 new group member in LGG 478 (along with 7 background galaxies). Only 4 of these new detections were not evident in the HIPASS data (Barnes et al. 2001), however. All of these new detections have been confirmed with ATCA, and have $\mathrm{M}_{H I}=10^{7-9} M_{\odot}$. All of the detections have associated stellar components; they are all dwarf galaxies.

Our survey found that the "missing satellite" problem is actually worse in the groups we surveyed than in the Local Group. Figure 1 shows that if we were observing the Local Group, we would detect a total of 14 galaxies, 9 of which have FWHM $\leq 100 \mathrm{~km} \mathrm{~s}^{-1}$ and could be considered dwarf galaxies. Data for the Local Group galaxies comes from Mateo (1998). The dwarf-to-giant ratio is 1.45 for the three groups we studied, compared with 1.8 for the Local Group. This discrepancy will be verified when we finish analyzing the remaining three groups in our sample, but, at the present, it appears that the Local Group is not unique in its deficit of luminous satellite galaxies compared to cold dark matter models of galaxy formation. 


\section{The Nature of High-Velocity Clouds}

HVCs are clouds of $\mathrm{H}$ I seen all around the Milky Way, but which are not in regular galactic rotation (see Wakker, these proceedings for a review). As such, their distances and masses are unknown, making their origins uncertain. Some HVCs are certainly associated with the Magellanic Stream which is the result of the tidal interaction between the LMC, SMC, and Milky Way, and it is possible that other complexes have similar origins (e.g. Lockman 2003). Other HVCs may be ejected material from a Galactic fountain, or may be primordial gas falling into the Local Group and onto the Milky Way as part of ongoing galaxy formation.

While the idea of HVCs being associated with galaxy formation is an old one, interest in the idea was revived by Blitz et al. (1999) and Braun \& Burton (1999) who suggested that HVCs and compact HVCs (CHVCs) may contain dark matter, have masses of $\sim 10^{7} M_{\odot}$, and reside at distances of $\sim 1 \mathrm{Mpc}$ from the Milky Way. These HVCs may even be associated with the large population of dark matter halos seen in the simulations discussed above. This idea has been expanded on by de Heij, Braun, \& Burton (2002b), who proposed that CHVCs were concentrated around the Milky Way and M31 with a Gaussian distance distribution of width $150-200 \mathrm{kpc}$, and $M_{H I} \leq 10^{7} M_{\odot}$. If CHVCs are associated with galaxy formation, then some of their analogs should be visible in the groups we have observed.

Starting with this premise, we have constructed a simple model for HVCs to predict how many should be seen in the groups we have studied. We begin by taking the integrated fluxes and velocity widths of all Milky Way CHVCs cataloged by Putman et al. (2002) and de Heij, Braun, \& Burton (2002a). We then randomly determine a distance assuming a Gaussian distance distribution of a given half-width, half-maximum. Given this distance, we get an H I mass for each cloud. Now we ask if this CHVC were in one of our groups, would we detect it at a $10 \sigma$ level. We do this for every CHVC to determine the number of expected detections per group for a given $\mathrm{D}_{H W H M}$, and then we repeat the process 100,000 times to get a distribution of expected detections. The results of these trials for two groups, LGG 93 and LGG 478, and two $\mathrm{D}_{H W H M}$ values are shown in Figure 2.

As we already stated in Section 3, we have found no HVC analogs ( $\mathrm{H} \mathrm{I}$ clouds without stars) in any of our groups. Therefore, in looking at Figure 2, we want to know for a given $\mathrm{D}_{H W H M}$ what the probability of zero detections is. Since this is a function of the mass detection limit of the group, we get different limits on $\mathrm{D}_{H W H M}$ for each group. For LGG 93, we can see in Figure 2 that $\mathrm{D}_{H W H M}$ is less than $400 \mathrm{kpc}$ at a $99.76 \%$ confidence level, but $\mathrm{D}_{H W H M}<250$ kpc is only $73 \%$ likely. For LGG 478 , the figure demonstrates that $\mathrm{D}_{H W H M}<$ $400 \mathrm{kpc}$ is $99.997 \%$ likely, and $\mathrm{D}_{H W H M}<250 \mathrm{kpc}$ is $93.8 \%$ certain. And for LGG 180 , not shown in the figure, the limits are $99.98 \%$ and $89 \%$ certain, respectively.

There are a couple of notes of caution regarding this analysis. First, it is important to note that the vast majority of CHVC analogs are not detected; we can only detect the most massive analogs. This means that we are using a small number of objects to infer the properties of a larger population. If the population of CHVCs in these groups is different than in the Local Group, we may not be able to detect any analogs. We also assume that the same number of 

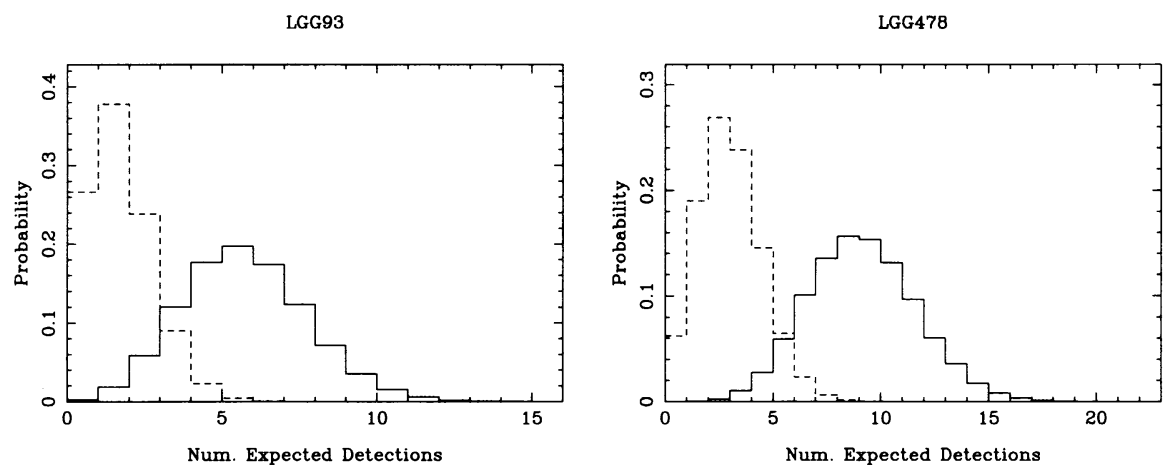

Figure 2. The probability of a given number of detections for LGG 93 (left) and LGG 478 (right). The solid line represents the probability distribution for $\mathrm{D}_{H W H M}=400 \mathrm{kpc}$ and the dashed line represents $\mathrm{D}_{H W H M}=250 \mathrm{kpc}$.

CHVCs is present in each group. If the number of CHVCs is a function of group mass, for example, we would expect less CHVCs in LGG 478 than in LGG 93 or LGG 180, so the constraints on $\mathrm{D}_{H W H M}$ may not be as strong as suggested above. Nevertheless by analogy to other groups, it appears that CHVCs should be clustered with $\mathrm{D}_{H W H M} \leq 300-400 \mathrm{kpc}$ of the Milky Way. At these distances, the total $\mathrm{H}$ I mass in CHVCs is only $\sim 10^{8} M_{\odot}$, making them an important source of fuel for star formation, but not dynamically important to the Local Group.

\section{Conclusions}

We have surveyed $\sim 1 \mathrm{Mpc}^{2}$ around the centers of 3 loose groups of galaxies using the Parkes Multibeam and the ATCA. The goal of these observations is to get a census of the H I-rich galaxies in these groups and to search for analogs to HVCs. This will permit us to constrain models for the origin of HVCs and to begin to test models of galaxy formation. These groups were chosen to be analogs to the Local Group, so that they only contain a few large spiral galaxies which are separated by a few hundred kiloparsecs. Our observations have very high velocity resolution, $<3.3 \mathrm{~km} \mathrm{~s}^{-1}$, in order to facilitate the detection of low-mass objects, so that our $1 \sigma$ detection limit is $\sim 7 \times 10^{5} M_{\odot}$ per $3.3 \mathrm{~km} \mathrm{~s}^{-1}$. Using fake sources to determine our completeness and ATCA observations to confirm our detections, we are roughly $100 \%$ complete and reliable at $10 \times$ the theoretical noise limit.

We found 7 new $\mathrm{H}$ I-rich objects in the groups, plus 7 background galaxies, only 4 of which were not seen in HIPASS, and all of which have stellar counterparts. The ratio of low-mass to high-mass galaxies in these groups is less than expected from simulations and also less than the ratio in the Local Group, therefore the "missing satellite" problem is not unique to our local neighborhood.

We found no analogs to HVCs in any of the three groups. Assuming the HVCs in these groups are the same as in the Local Group, this implies that compact HVCs must be clustered within $\sim 300-400 \mathrm{kpc}$ of the Milky Way, oth- 
erwise we would have seen their analogs in our survey. This is strong evidence against the Blitz et al. (1999) and Braun \& Burton (1999) models for the origin of HVCs, but is still consistent with the de Heij et al. (2002b) model. At these distances, however, the total $\mathrm{H}$ I mass in CHVCs is only $\sim 10^{8} M_{\odot}$, making them unimportant to the dynamics of the Local Group, but still a useful reservoir of fuel for future star formation.

We have Parkes data in hand for an additional 3 groups, and will be following up our detections in these groups with the ATCA and the VLA in the near future. The ensemble of the data on these 6 groups will place stronger constraints on the nature of HVCs and provide a better test of different models of galaxy formation.

Acknowledgments. The authors wish to thank the excellent staff at Parkes and the ATCA for their assistance with observing. We wish to thank Martin Zwaan for his assistance with inserting fake sources into our data cubes. D.J.P. acknowledges generous support from NSF MPS Distinguished Research Fellowship grant AST0104439.

\section{References}

Barnes, D.G. 2001, MNRAS, 322, 486

Blitz, L., Spergel, D., Teuben, P.J., Hartmann, D., Burton, W.B. 1999, ApJ, 514,818

Braun, R., Burton, W.B. 1999, A\&A, 351, 437

de Heij, V., Braun, R., Burton, W.B. 2002a, A\&A, 391, 67

de Heij, V., Braun, R., Burton, W.B. 2002b, A\&A, 392, 417

Garcia, A.M. 1993, A\&AS, 100, 47

Klypin, A.A., Kravtsov, A.V., Valenzuela, O., Prada, F. 1999, ApJ, 522, 82

Lockman, F.J. 2003, ApJ, 591, L33

Mateo, M. 1998, ARA\&A, 36, 435

Moore, B., Ghigna, S., Governato, F., Lake, G., Quinn, T., Stadel, J. 1999, ApJ, 524, L19

Putman, M.E., et al. 2002, AJ, 123, 873

Tully, R.B. 1987, ApJ, 321, 280

Zabludoff, A.I., Mulchaey, J.S. 1998, ApJ, 498, L5 